\title{
Bullous Systemic Lupus Erythematosus in a Pregnant Woman associated with Lupus Nephritis Responding to Dapsone
}

\author{
Vinicki JP${ }^{1 *}$, Juarez MA², Muzzio I ${ }^{3}$, Dubinsky $D^{1}$, Mazzocchi ${ }^{4}$, Risso JA ${ }^{4}$, \\ Nasswetter $\mathbf{G}^{1}$ \\ ${ }^{1}$ Division Rheumatology, Hospital das Clinicas, University of Buenos Aires, Argentina \\ ${ }^{2}$ Department of Pathology, Hospital de Clinicas, University of Buenos Aires, Argentina \\ ${ }^{3}$ Division Dermatology, Hospital das Clinicas, University of Buenos Aires, Argentina \\ ${ }^{4} 5$ th Chair, Department of Medicine, Hospital de Clinicas Jose de San Martin, University of Buenos Aires, Argentina
}

Received: July 25, 2016; Accepted: July 26, 2016; Published: August 2, 2016

*Corresponding author: Juan Pablo Vinicki, Division Rheumatology, Hospital das Clinicas, Avenida Córdoba 2351 (C1120AAR), Buenos Aires, Argentina, Email: jpvinicki@hotmail.com

\begin{abstract}
Bullous systemic lupus erythematosus (b-SLE) is a rare blistering condition with a distinctive combination of clinical, histological and immune pathologic features that together constitute a unique bullous disease phenotype. It is often associated with autoimmunity to type VII collagen. Here we report a 23 years old woman with an 11-week pregnancy diagnosed with lupus six months prior to consultation who presented with b-SLE associated with renal disease. Rapid resolution of the blisters occurred following treatment with dapsone. This is the first report of b-SLE during pregnancy associated with lupus nephritis in English literature.
\end{abstract}

\section{Introduction}

Systemic lupus erythematosus (SLE) is an autoimmune disease characterized by the involvement of multiple organs in the context of the production of a variety of auto-antibodies. Clinical manifestations are variable, being the cutaneous involvement in $76 \%$ of patients in the course of the disease.

Bullous SLE (b-SLE) is a subepidermal blistering disease that occurs in less than $5 \%$ of patients with SLE [1]. Clinically, patients present with the rapid, widespread development of tense fluid-filled vesicles and bullae, often on a background of erythema and urticaria. The lesions can affect any area of the body, including mucous membranes, but tend to favor the upper trunk, proximal extremities, neck, and face. In addition, patients with SLE may have concurrent bullous disease like pemphigus, dermatitis herpetiformis, bullous pemphigoid, epidermolysis bullosa acquisita and linear IgA bullous disease.

In English literature, we found only one case of b-SLE during pregnancy but without nephritis [2]. We report a case of a patient with b-SLE and lupus nephritis in early pregnancy.

\section{Clinical Case}

A 23 years old woman with an 11-week pregnancy diagnosed with SLE six months ago (malar rash, photosensitivity, oral ulcers, arthritis, positive ANA 1/2560 with a homogeneous pattern, positive anti-DNA, anti-sm and hypocomplementemia) complained of blisters on skin and mucous membranes lasting more than 1 week. The patient was under treatment with hydroxylchloroquine $400 \mathrm{mg} /$ day and meprednisone $8 \mathrm{mg} /$ day. Physical examination revealed urticarial, erythematous eruption associated with tense blisters, erosions, and crusting over face, trunk, extremities and flexures of the arm and groin regions as well asoral and genital mucosa Fig 1\&2. Some of these bullae arose on a background of normal appearing skin.Antenatal history was normal (one previous normal pregnancy).

Laboratory showed anemia of chronic disease, lymphopenia $(1100 / \mathrm{ml})$, dysmorphic hematuria with RBC casts associated with nephrotic range proteinuria (5.05 g/24 hours) without impaired renal function with positive anti-DNA, anti-Ro, anti-La, anti-RNP and decreased complement with negative lupus inhibitor. Skin

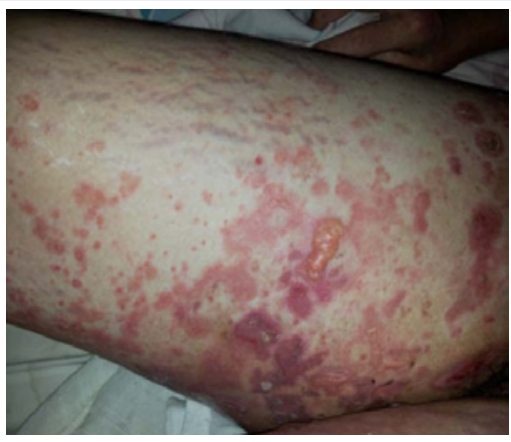

Figure 1: Serous blisters content in thigh and skin wounds secondary to ruptured blister. 
biopsy displayed subepidermal blister with neutrophils and perivascular lymphocytic infiltrate in dermis. Direct immune fluorescence showed a lineal band of IgG and granular deposit of $\operatorname{IgA}$ at the basement membrane Fig 3 .

Considering the cutaneous involvement associated with renal disease during pregnancy, treatment was started with meprednisone $1 \mathrm{mg} / \mathrm{kg} /$ day and azathioprine $2 \mathrm{mg} / \mathrm{kg} /$ day. After 4 weeks of treatment, improvement of renal disease was observed but bullous disease did not ameliorate. Due to worsening of skin lesions, dapsone $100 \mathrm{mg}$ /day was started (16-week pregnancy). At 48 hours, absence of new blisters was observed and complete skin healing took 14 days.

During hospitalization, obstetric controls (fetal ultrasound with Doppler and echocardiogram) were normal without affecting growth or weight during the entire hospitalization. Due to the favorable clinical course, the patient (in 18-week pregnancy)

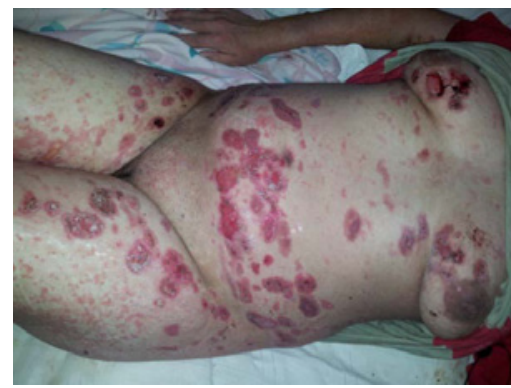

Figure 2: Skin wounds in chest, abdomen and thighs due to ruptured blister.

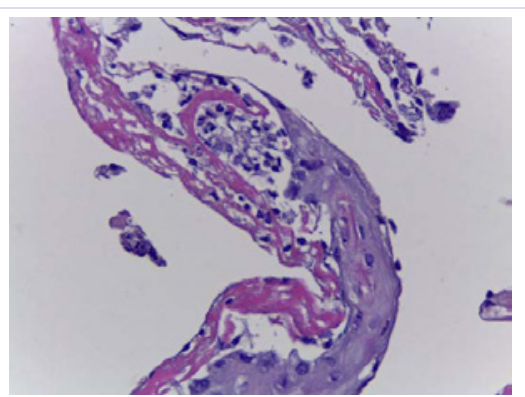

Figure 3: Sub-epidermal blister on skin biopsy (H\&E, 400X).

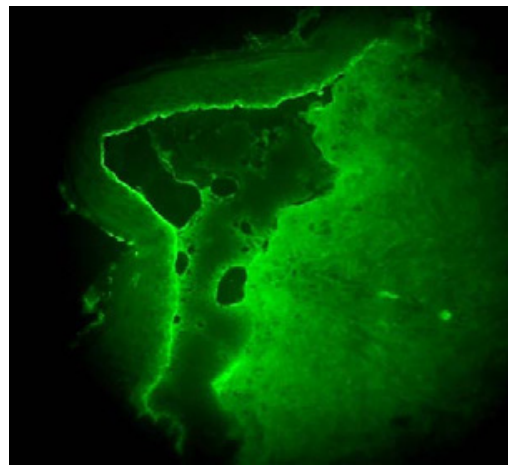

Figure 4: Immuno fluorescence: linear deposit of IgG. was discharged with scheduled outpatient visits. Unfortunately, it was lost to follow up and no data regarding postpartum and newborn is available.

\section{Discussion}

It is believed that the b-SLE is caused by auto-antibodies against type VII collagen. The latter is an important component of the anchoring fibrils that maintain adhesion at the dermalepidermal junction by cross-links between the lamina densa and the matrix of the papillary dermis; in this location, interference between type VII collagen and their ligands results in defective adherence [3]. In vitro studies have shown that collagen type VII antibodies have the potential to activate complement and generate peptides that stimulate proteolysis mediated by neutrophil causing detachment at the dermo-epidermal junction [4].

Camisa and Sharma first proposed diagnostic criteria for b-SLE in 1983, which were later revised after the administration of salt split-skin immune fluorescence [5]. The diagnosis of b-SLE is based on the following five criteria: vesiculobullous rash, biopsy confirmation of subepidermal blister with neutrophil predominance, evidence in direct immune fluorescence of IgG in the basement membrane of the dermis (the deposits can be linear or granular with or without IgA and/or IgM), evidence of type VII collagen auto-antibodies (by indirect immune fluorescence) and good response to treatment with dapsone [6].

Pregnancy may increase disease activity and precipitate the appearance of flares (13\% to $75 \%$ ) in SLE, which are usually mild and the most frequently affected organs are the skin and joints [7]. Lupus pregnancies are considered high risk for maternal and fetal complications. A recent meta-analysis reviewing 2571 pregnancies in SLE patients reported unsuccessful pregnancy and preterm birth rates of $23 \%$ and $39 \%$, respectively, while maternal complications included nephritis (16\%), hypertension (16\%), and pre-eclampsia (7\%) [8].Controversy exists between the presence of blisters and renal involvement. However, most authors found bullous disease that coincided with disease activity [9- 11]. In fact, Malcangi et al described association between bullous eruption and clinical or serologic relapse in 9 of 25 cases (26\%) [12]. In addition, 4 patients had renal involvement.

One important differential diagnosis in the context of bullous diseases in pregnancy is pemphigoidgestationis (PG). The clinical presentation is very similar to b-SLE but under light microscopy there is often papillary oedema with eosinophilic spongiosis and subsequent subepidermal bullae formation [13]. On direct immune fluorescence, PG is characterized by linear deposition of $\mathrm{C} 3$, and occasionally IgG, along the basement membrane zone of peri lesional skin. Frequently, indirect immune fluorescence demonstrates a circulating IgG1 autoantibody (PG factor), directed against a component of the hemidesmo some of the basement membrane zone which avidly binds complement [13]. Pregnancy does not seem to be a risk factor for developing b-SLE as there are only 2 cases published to date (including our case).

b-SLE does not usually respond to systemic corticosteroid 
treatment, as with other clinical manifestations in lupus. Dapsone (category C during pregnancy) is the mainstay of treatment [14]. Rapid response can be obtained by stopping the development of new blisters in 24-48 hours and healing of existing injuries is achieved later. Relapse might occur in case of discontinuation, although rapid response after restarting therapy is observed. Discontinuation of treatment is possible within 12 months. To reduce the risk of hemolysis, it is recommended not to exceed $1.5 \mathrm{mg} / \mathrm{kg} /$ day.

In summary, b-SLE is a rare skin manifestation in these patients. Accurate assessment of disease activity and flares plus stratification according to severity of target organ involvement should be considered (especially lupus nephritis). Patients usually do not respond to systemic corticosteroids or immune suppression and dapsone is the treatment of choice.

\section{Conflict of Interest}

This research received no specific grant from any funding agency in the public, commercial, or not-for-profit sectors.

\section{References}

1. Dhir R, Desylva P, Gehi N , Malik A, Singh YD, Jagannayakulu H, et al Pericardial effusion with vesiculobullous lesions in a young female. Bullous systemic lupus erythematosus (bullous SLE). Indian J Dermatol Venereol Leprol. 2006;72:175-177.
2. Dos Santos CE, Velho PH, Marques FM, Werner B, Aragão SC, Rachid Filho A. Bullous systemic lupus erythematosus in a pregnant woman: a case report. Rev Bras Reumatol. 2013;53(5):438-440.

3. Gammon WR. Autoimmunity to collagen VII: autoantibody-mediated pathomechanisms regulate clinico-pathological phenotypes of acquired epidermolysis bullosa and bullous SLE. J Cutan Path. 1993;20(2):109-114.

4. Gammon WR, Briggaman RA, Inman AO III, Merritt CC, Wheeler CE Jr. Evidence supporting a role for immune complex mediated inflammation in the pathogenesis of bullous lesions of systemic lupus erythematosus. J Invest Dermatol. 1983;81(4):320-325.

5. Camisa C, Grimwood RE. Indirect immune fluorescence in vesciculobullous eruption of systemic lupus erythematosus. J Invest Dermatol. 1986;86(5):606.

6. Tincopa M,Puttgen KB, Sule S, Cohen BA, Gerstenblith MR. Bullouslupus: an unusualinitial presentation of systemic lupus erythematosus in an adolescent girl. Pediatr Dermatol. 2010;27(4):373-376. doi:10.1111/ j.1525-1470.2010.01179.x.

7. Al Arfaj AS, Khalil N. Pregnancy outcome in 396 pregnancies in patients with SLE in Saudi Arabia. Lupus. 2010;19(14):16651673. doi:10.1177/0961203310378669.

8. Smyth A, Oliveira GH, Lahr BD, Bailey KR, Norby SM, Garovic VD. 